\title{
Antibiotic-Induced Increase in Inflammatory Markers in Cured Infectious Spondylitis : Two Case Reports
}

\author{
Ki Seong Eom \\ Department of Neurosurgery, Wonkwang University School of Medicine, Iksan, Korea
}

Conservative therapy with appropriate antibiotics is essential for most patients with infectious spondylitis. Although most antibiotics do not cause problems if it used properly and serious side effects are rare, side effects can occur with any class of drugs and adverse reactions of antibiotics can range from mild allergic reactions to serious and fulminant adverse events. These side effects are also extremely variable from patient to patient and from antibiotic to antibiotic. A side effect of antibiotics may paradoxically increase inflammatory marker levels. Here, the author presents two cases of antibiotic-induced increase in inflammatory markers in cured infectious spondylitis. The patients were successfully treated after stopping the antibiotic therapy. The differential diagnosis between antibiotic side effects and infection should be considered very carefully because the treatment is completely different. Although the exact mechanisms underlying successful treatment without antibiotics are unclear, we should consider the side effects of antibiotics when following inflammatory markers during treatment of infectious spondylitis.

Key Words : Antibiotics · Spondylitis · Inflammation · Infection.

\section{INTRODUCTION}

In addition to clinical symptoms and signs, radiologic and laboratory investigation is commonly used to support the diagnosis and assessment of an inflammatory process ${ }^{7}$. The erythrocyte sedimentation rate (ESR) and C-reactive protein (CRP) level are useful for screening and assessment in the diagnosis and treatment of infectious spondylitis. Compared to the ESR, CRP is a more sensitive and specific acute phase reactant and is more responsive to changes in the course of infection ${ }^{6,13)}$. However, an elevation in CRP and/or ESR should not be taken as pathognomonic for infection ${ }^{7)}$.
Conservative therapy with appropriate antibiotics is essential for most patients with infectious spondylitis ${ }^{13}$. With resolution of infectious spondylitis, the CRP and ESR levels should decrease ${ }^{6,7)}$. If these inflammatory markers remain consistently elevated despite use of intravenous (IV) antibiotics, a repeat radiological study should be considered to look for any infectious lesions that may require a longer course of IV therapy ${ }^{5,13)}$. However, side effects can occur with any class of drugs and adverse reactions of antibiotics can range from mild allergic reactions to serious and fulminant adverse events. These side effects are also extremely variable from patient to patient and from antibiotic to antibiotic ${ }^{17}$. A side effect of antibiotics is a

- Received : March 8, 2018 •Revised : April 21, 2018 •Accepted : May 18, 2018

- Address for reprints : Ki Seong Eom

Department of Neurosurgery, Wonkwang University College of Medicine, 895 Muwang-ro, Iksan 54538, Korea

Tel : +82-63-859-1462, Fax : +82-63-852-2606, E-mail : kseom@wonkwang.ac.kr, ORCID : https://orcid.org/0000-0002-8354-4024

This is an Open Access article distributed under the terms of the Creative Commons Attribution Non-Commercial License (http://creativecommons.org/licenses/by-nc/4.0) which permits unrestricted non-commercial use, distribution, and reproduction in any medium, provided the original work is properly cited. 
paradoxical increase in inflammatory marker levels despite cure of infection. The author presents two cases of antibioticinduced increase in inflammatory markers in cured infectious spondylitis. The patients were successfully treated after stopping the antibiotic therapy.

\section{CASE REPORT}

\section{Case 1}

A 67-year-old man was admitted with pain and numbness in the thighs, buttocks, and calves, precipitated by walking. Magnetic resonance imaging (MRI) revealed spinal stenosis at L4/5 with hypertrophy of the ligamentum flavum. He underwent mini-open posterior lumbar interbody fusion with percutaneous screw fixation and was discharged on the 9th postoperative day (POD). However, he returned to our hospital because he experienced severe lower back pain and fever on the 18th POD. Laboratory investigation revealed ESR of 52 $\mathrm{mm} / \mathrm{h}$ (normal range, $0-20$ ), CRP concentration of $66.04 \mathrm{mg} / \mathrm{L}$ (normal range, $0-5$ ), white blood cell (WBC) count of 11600/ $\mathrm{L}$ (normal range, 4000-10000/ $\mu \mathrm{L}$ ), neutrophil count of $9270 / \mu \mathrm{L}$ (normal range, 1500-7500/ $\mu \mathrm{L}$ ), and eosinophil count of $80 / \mu \mathrm{L}$ (normal range, 40-500/ $\mu \mathrm{L}$ ). Lumbar contrast-enhanced computed tomography (CT) revealed postoperative infection with abscess formation along the screw heads and rods (Fig. 1A). We performed aspiration and irrigation of the abscess under local anesthesia. Staphylococcus caprae was cultured from the abscess, and IV vancomycin was started based on the result of antimicrobial sensitivity testing. We continued IV antibiotics therapy for 2 months, following which the ESR gradually decreased to $16.1 \mathrm{~mm} / \mathrm{h}$ and the CRP to $10.52 \mathrm{mg} / \mathrm{L}$. All other laboratory investigations also were normal. The inflammatory markers subsequently increased rapidly, with an ESR of 45 $\mathrm{mm} / \mathrm{h}$ and CRP of $62 \mathrm{mg} / \mathrm{L}$, although his severe lower back pain was relieved. Eosinophil count has also markedly increased to $6130 / \mu \mathrm{L}$. Lumbar enhanced CT showed nearly complete disappearance of the abscess (Fig. 1B). At that time, the patient complained of whole-body pruritus and skin rash. We suspected an adverse effect and drug eruption and stopped the antibiotic. One week later, his laboratory tests had rapidly returned to normal with an ESR of $10 \mathrm{~mm} / \mathrm{h}$ and CRP of 2.17 $\mathrm{mg} / \mathrm{L}$ and his skin symptoms completely disappeared. Eosinophil count was still increased to 5260 and normalized after 3 weeks (Fig. 1C).

\section{Case 2}

An 82-year-old man was transferred from another hospital for further evaluation and treatment. Approximately 4 months before transfer, he underwent several lumbar epidural blocks for low back pain. One month after these procedures, his low back pain worsened and developed a fever. Laboratory investigations revealed an increased WBC count of 13700/ $\mu \mathrm{L}$, ESR of $93 \mathrm{~mm} / \mathrm{h}$, and CRP of $51.0 \mathrm{mg} / \mathrm{L}$. Lumbar MRI at another hospital suggested infectious spondylitis at L4 and L5 (Fig. 2A). He underwent IV antibiotic therapy with moxifloxacin and cefixime. During 2 months of IV antibiotic therapy, low back pain was relieved and the fever and inflammatory makers steadily decreased. The inflammatory markers subsequently increased rapidly, and additional IV antibiotic therapy
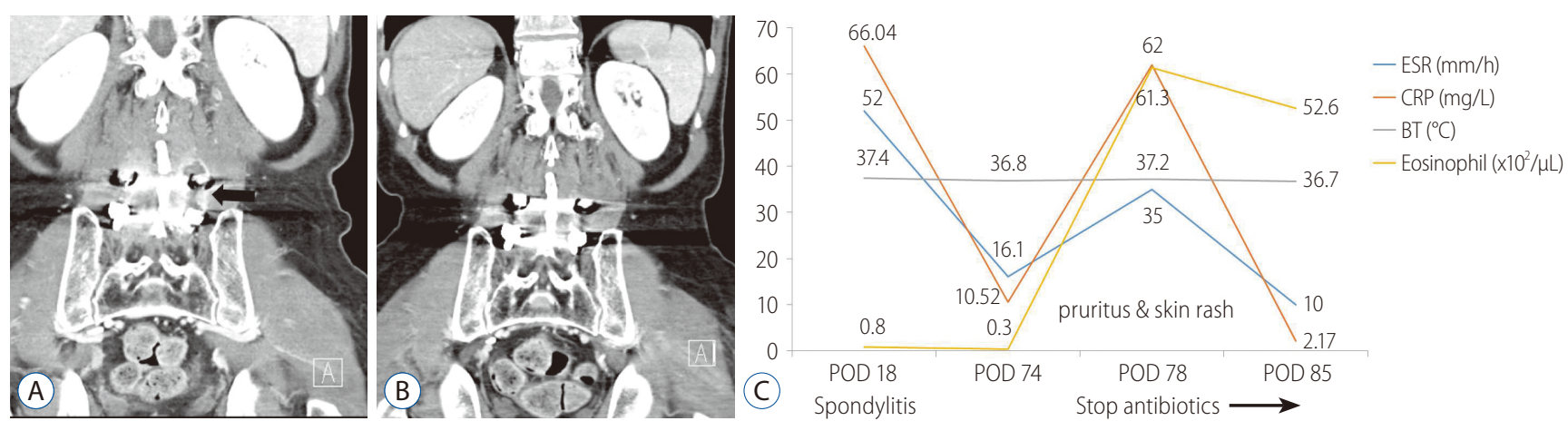

Fig. 1. Case 1. A : Lumbar coronal contrast-enhanced CT showing postoperative infection with abscess formation along the screw heads and rods (black arrow) before antibiotics therapy. B : CT obtained after antibiotic therapy for 2 months showing complete disappearance of abscess. C : The course of inflammatory markers with ESR and CRP, BT, and eosinophil. ESR : erythrocyte sedimentation rate, CRP : C-reactive protein, BT : body temperature, $\mathrm{POD}$ : postoperative day, CT : computed tomography. 
for suspected relapse of infectious spondylitis was recommended. However, the patient's family rejected the recommendation and requested a transfer to the university hospital. Laboratory investigation on admission to our hospital revealed a ESR $41 \mathrm{~mm} / \mathrm{h}$, CRP of $27.15 \mathrm{mg} / \mathrm{L}$, WBC count of $9400 / \mu \mathrm{L}$, neutrophil count of $6400 / \mu \mathrm{L}$, and eosinophil count of $170 / \mu \mathrm{L}$. A three-phase bone scan with Tc-99m 2,3-dicarboxypropane-1,1-diphosphonate was compatible with active spondylodiscitis at L4 and L5 (Fig. 2B). We suspected an adverse drug effect and recommended stopping antibiotic therapy because he had abdominal pain with diarrhea, the inflammatory markers were not extremely elevated, the pain was not manageable, and there was no fever. We advised his family that we would perform isolation and microbial culture and resume antibiotic therapy if the inflammatory markers increased after stopping the antibiotics. Two weeks after stopping the antibiotics, the all laboratory tests returned to normal. His pain resolved and he was discharged 2 weeks after admission.

\section{DISCUSSION}

Infectious spondylitis is defined as an infection of one or more components of the spine by a specific organism ${ }^{7)}$. Most patients with infective spondylitis can be treated conservatively with antibiotics ${ }^{5,6)}$. Because the clinical symptoms vary widely in the early stages, it is difficult to differentiate infectious spondylitis from other diseases, resulting in delayed diagnosis or misdiagnosis. The onset of symptoms is commonly insidious, with spinal pain being the most common presenting complaint. Although more than $90 \%$ of cases are pyogen$\mathrm{ic}^{6,9)}$, fever is typically not present and occurs in less than $20 \%$ of patients ${ }^{6,13)}$.

The WBC count, ESR, and CRP are indicators of inflammation in patients with infectious spondylitis. The WBC count is elevated in $40-66 \%$ of patients, and is not particularly useful in making a diagnosis of infectious spondylitis ${ }^{13)}$. The ESR is a sensitive indicator of infectious spondylitis, and is positive in $76-81 \%$ of patients at the time of diagnosis, with levels ranging from $43-87 \mathrm{~mm} / \mathrm{h}$ in pyogenic spondylitis ${ }^{4,13)}$. Elevation of ESR is correlated with the inflammatory response but is not specific for infection. The ESR is often significantly affected by many factors other than the acute phase reaction ${ }^{7}$, including the plasma albumin level; the size, shape, and number of red blood cells; and non-acute phase reaction proteins, in particular normal and abnormal immunoglobulins. The lack of specificity of the ESR means the test is more likely to be falsely positive than the CRP. Moreover, the slow response of ESR to the acute phase reaction leads to false negatives early in an inflammatory process ${ }^{8)}$. Despite the nonspecific nature of an elevation in the ESR, this test provides additional data regarding the possible presence of infection and some information on the response to treatment. CRP is a useful marker of the acute phase reaction as it responds quickly to the inflammatory process ${ }^{7,11)}$. It is an acute phase protein synthesized by hepatocytes. Although CRP activation of complement increases inflammation and tissue damage, it also has some anti-inflammatory activity, and acts as a promoter and down-regulator of inflammation ${ }^{11)}$. CRP is elevated in $90 \%$ or

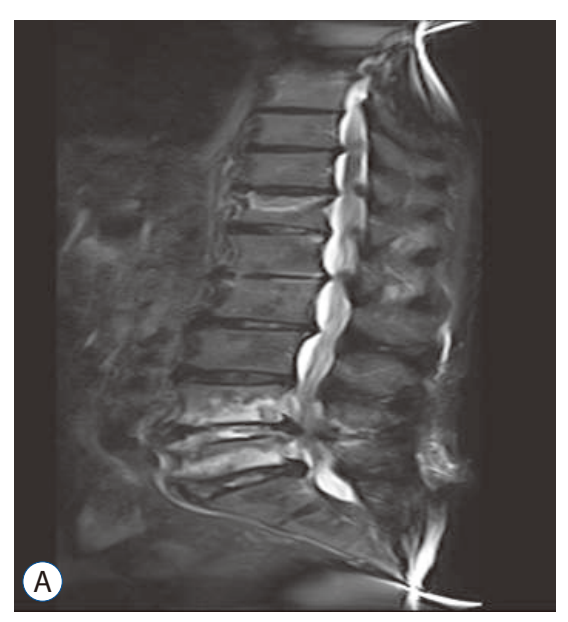

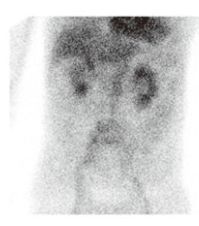

ANT 5 MIN

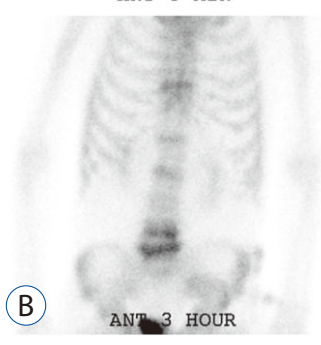

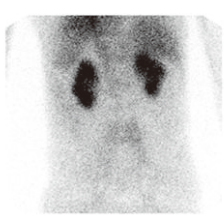

POST 5 MIN

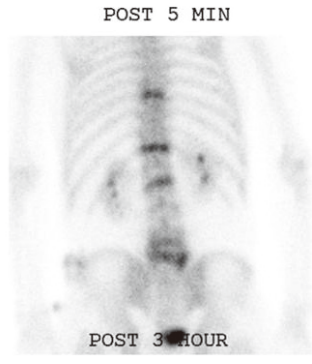

Fig. 2. Case 2. A : Sagittal fat-suppressed T2-weighted image showing infectious spondylitis at L4 and L5. B : A three-phase bone scan shows active spondylodiscitis at L4 and L5. 
more of patients with spinal infection, but is more specific than ESR and normalizes postoperatively or after appropriate treatment of an infectious process, more rapidly than the $\mathrm{ESR}^{7,11}$. A majority of patients with infective spondylitis can be treated without surgery. Although the optimal duration is not well defined, several studies recommend 6-8 weeks of IV antibiotics and others recommend only 4 weeks ${ }^{5,6,13)}$. Insufficient IV antibiotic therapy for less than 4 weeks may result in a high recurrence rate. Some authors advise use of IV antibiotics until the CRP is normal ${ }^{6}$.

Antibiotics are used for the treatment and prevention of many infectious disorders and are thought to be safe when applied properly. However, like all drugs, they also show various adverse effects in some patient conditions ${ }^{17)}$. Antibiotic complications can affect the hematologic, cardiac, respiratory, gastrointestinal, hepatobiliary, renal, genitourinary, rheumatologic, dermatologic, and neurologic systems, and can cause a drug fever ${ }^{17)}$. Multiple antibiotics are associated with QT prolongation and may increase the risk of sudden cardiac arrest due to torsades de pointes ${ }^{10)}$. Patients treated with antibiotics frequently experience diarrhea. Although Clostridium difficile infection is obviously of great concern, the majority of diarrhea cases will not be attributable to this infection. Nausea is frequently encountered and it is often difficult to identify a specific cause, although it should be noted that there are some antibiotics for which this is a very common side effect ${ }^{3)}$. The most common dermatologic adverse reaction associated with antibiotic therapy is a drug-induced exanthem, or "drug rash." Parenteral therapy with beta-lactams (penicillins and cephalosporins) is associated with drug-induced fever, as is therapy with several other antibiotics including sulfonamides ${ }^{15}$.

A research carried out at six pharmacovigilance centers in Korea reported that antibiotics including vancomycin were the most frequent causes of adverse reactions of drug, and that cutaneous symptoms were the most common manifestations in adverse reactions ${ }^{12}$. In this study, 3.1\% of 1418 cases were associated with vancomycin. An et at. ${ }^{1)}$ reported that the skin rashes associated with increased peripheral eosinophil, representing suspected immune-mediated delayed hypersensitivity reactions, are a common adverse reaction of vancomycin. Eosinophilia is considered when absolute eosinophil count exceeds 500/ $\mu \mathrm{L}$ in peripheral blood. Peripheral eosinophilia can be caused by parasitic infections, allergy, drug reactions, leukemia, and non-hematologic malignancies ${ }^{14)}$. The author treated infectious spondylitis with vancomycin in case 1 patient. During the period of treatment with vancomycin, inflammatory markers decreased gradually. However, after 2 months, the inflammatory markers subsequently increased rapidly, and whole-body pruritus and skin rash were observed Laboratory tests revealed significant eosinophilia. The author suspected that an adverse effect of allergic reaction of adverse drug effects.

Case 2 patient treated with moxifloxacin and cefixime had no eosinophilia or skin symptoms other than abdominal pain due to diarrhea. Antibiotic-associated diarrhea is defined as unexplained diarrhea association with antibiotic administration $^{3)}$. Although the frequency of antibiotics-associated diarrhea depends on the antibiotics, it occurs in approximately 5.2 to $6.2 \%$ of patients who are treated with moxifloxacin and 15 to $20 \%$ of those who receive cefixime ${ }^{2,3}$. A common first step is to identify cases of antibiotic-associated diarrhea that are due to Clostridium difficile infection, because this is the most common identifiable and treatable pathogen. The tests used for diagnosis depend on the type of laboratory investigations available. Enzyme immunoassays for detecting toxin A or toxins A and B are generally available ${ }^{3}$. In case 2, Clostridium difficile toxin was not detected. Only $10-20 \%$ of the stool specimens submitted for testing of Clostridium difficile toxins are reported as positive ${ }^{16)}$. Antibiotic-associated diarrhea can also be caused by other enteric pathogens, direct effects of antibiotics on the intestinal mucosa, and the metabolic consequences of reduced concentrations of fecal flora. Many patients with enteric disease caused by antibiotics have a response to withdrawal of the inducing agent ${ }^{3,16}$. The author thought that a direct effect of antibiotics affected the digestive system has increased levels of inflammatory marker in case 2 patient. After stopping antibiotics, the inflammatory markers in both cases rapidly returned to normal.

\section{CONCLUSION}

As with any medication, antibiotics can cause side effects. Most antibiotics do not cause problems if it used properly and serious side effects are rare. It is possible that the inflammation due to these antibiotic complications may result in a greater increase in the inflammatory markers. The differential diagnosis between antibiotic side effects and infection should 
be considered very carefully because the treatment is completely different. We should identify the systemic symptoms of patient and closely monitor laboratory tests including complete blood counts for accurate detection of the side effects of antibiotics when following inflammatory markers during treatment of infectious spondylitis. The antibiotics cannot be effective and the inflammation may not be well controlled when the elevation of the inflammatory marker is accompanied by eosinophilia or diarrhea. Further observations are necessary to clarify the clinical and laboratory significance of this phenomenon.

\section{CONFLICTS OF INTEREST}

No potential conflict of interest relevant to this article was reported.

\section{INFORMED CONSENT}

Informed consent was obtained from all individual participants included in this study.

\section{AUTHOR CONTRIBUTIONS}

\author{
Conceptualization : KSE \\ Data curation : KSE \\ Formal analysis : KSE \\ Funding acquisition : KSE \\ Methodology : KSE \\ Project administration : KSE \\ Visualization : KSE \\ Writing - original draft : KSE \\ Writing - review \& editing : KSE
}

\section{- Acknowledgements}

This work was supported by Wonkwang University in 2018.

\section{References}

1. An SY, Hwang EK, Kim JH, Kim JE, Jin HJ, Jin SM, et al. : Vancomycinassociated spontaneous cutaneous adverse drug reactions. Allergy Asthma Immunol Res 3 : 194-198, 2011

2. Ball $P$, Stahlmann $R$, Kubin $R$, Choudhri $S$, Owens $R$ : Safety profile of oral and intravenous moxifloxacin: cumulative data from clinical trials and postmarketing studies. Clin Ther 26 : 940-950, 2004

3. Bartlett JG : Clinical practice. Antibiotic-associated diarrhea. N Engl J Med $346: 334-339,2002$

4. Carragee EJ, Kim D, van der Vlugt T, Vittum D : The clinical use of erythrocyte sedimentation rate in pyogenic vertebral osteomyelitis. Spine (Phila Pa 1976) 22 : 2089-2093, 1997

5. Chelsom J, Solberg CO : Vertebral osteomyelitis at a Norwegian university hospital 1987-97: clinical features, laboratory findings and outcome. Scand J Infect Dis 30 : 147-151, 1998

6. Cheung WY, Luk KD : Pyogenic spondylitis. Int Orthop 36 : 397-404, 2012

7. Harrison $\mathrm{M}$ : Erythrocyte sedimentation rate and C-reactive protein. Aust Prescr 38 : 93-94, 2015

8. Osei-Bimpong A, Meek JH, Lewis SM : ESR or CRP? A comparison of their clinical utility. Hematology $12:$ 353-357, 2007

9. Park JH, Shin HS, Park JT, Kim TY, Eom KS : Differentiation between tuberculous spondylitis and pyogenic spondylitis on MR imaging. Korean J Spine 8 : 283-287, 2011

10. Ray WA, Murray KT, Hall K, Arbogast PG, Stein MC : Azithromycin and the risk of cardiovascular death. N Engl J Med 366 : 1881-1890, 2012

11. Reeves $G$ : C-reactive protein. Aust Prescr $30: 74-76,2007$

12. Shin YS, Lee YW, Choi YH, Park B, Jee YK, Choi SK, et al. : Spontaneous reporting of adverse drug events by Korean regional pharmacovigilance centers. Pharmacoepidemiol Drug Saf 18 : 910-915, 2009

13. Son DW, Song KS : Infectious spondylitis in Zhang HY (ed) : The Textbook of Spine, ed 2. Seoul : Koonja, 2013, pp966-977

14. Tefferi A : Blood eosinophilia: a new paradigm in disease classification, diagnosis, and treatment. Mayo Clin Proc 80 : 75-83, 2005

15. Usatine RP, Sandy N : Dermatologic emergencies. Am Fam Physician $82: 773-780,2010$

16. Wiström J, Norrby SR, Myhre EB, Eriksson S, Granström G, Lagergren L, et al. : Frequency of antibiotic-associated diarrhoea in 2462 antibiotictreated hospitalized patients: a prospective study. J Antimicrob Chemother 47 : 43-50, 2001

17. Wright J, Paauw DS : Complications of antibiotic therapy. Med Clin North Am 97 : 667-679, xi, 2013 